Fanum

Sociológico

\section{Forum Sociológico}

Série II

17 | 2007

Envelhecimento activo. Um novo paradigma

\title{
Envelhecimento activo e estilos de vida saudáveis : a actividade física
}

Paulo Ferreira Pinto, Ana Alexandre Fernandes e Maria Amália Botelho

\section{(2) OpenEdition \\ 1 Journals}

Edição electrónica

URL: https://journals.openedition.org/sociologico/1629

DOI: 10.4000/sociologico.1629

ISSN: 2182-7427

Editora

CICS.NOVA - Centro Interdisciplinar de Ciências Sociais da Universidade Nova de Lisboa

Edição impressa

Data de publição: 1 janeiro 2007

ISSN: 0872-8380

Refêrencia eletrónica

Paulo Ferreira Pinto, Ana Alexandre Fernandes e Maria Amália Botelho, «Envelhecimento activo e estilos de vida saudáveis : a actividade física», Forum Sociológico [Online], 17 | 2007, posto online no dia 01 janeiro 2007, consultado o 31 março 2022. URL: http://journals.openedition.org/sociologico/ 1629 ; DOI: https://doi.org/10.4000/sociologico.1629 


\title{
ENVELHECIMENTO ACTIVO E ESTILOS DE VIDA SAUDÁVEIS: A ACTIVIDADE FÍSICA
}

\author{
Paulo Ferreira Pinto \\ da FCM, UNL (paulo.ferpinto@terra.com.br) \\ Ana Alexandre Fernandes \\ Socióloga, Professora Associada (FCM, UNL) (ana.alexandre@fcm.unl.pt) \\ Maria Amália Botelho \\ Médica, Professora Auxiliar (FCM, UNL) (mabotelho.fisio@fcm.unl.pt)
}

Faculdade de Educação Física e Desportos da Universidade Federal de Juiz de Fora, Minas Gerais, Brasil e doutorando

\begin{abstract}
Resumo
Uma das grandes preocupações sobre a população mundial para os próximos 50 anos é o processo de envelhecimento da população, a sua repercussão sobre os problemas de saúde, a vigência de uma transição epidemiológica em concomitância com novas doenças, a busca de avanços científicos e tecnológicos para responder à procura emergente e a adopção e consolidação de modelos de saúde centrados na prevenção. A ênfase em novos paradigmas constitui ponto convergente das abordagens dos órgãos oficiais (inter)nacionais, havendo incentivo para a realização de investigações nas áreas biomédicas e nas ciências sociais. As políticas públicas implantadas têm como preocupação maximizar a melhoria da qualidade dos anos vividos da população, numa perspectiva interdisciplinar, integrada, articulada e individualizada, que percorra todo o curso de vida e seja capaz de consolidar estratégias de estilos de vida saudáveis, enfatizando, entre outras, a prática regular da actividade física.
\end{abstract}

Palavras-chave: envelhecimento, saúde pública, actividade física, estilos de vida saudáveis.

\begin{abstract}
One of the great concerns about global population for the next 50 years is the aging process, its repercussion on health, the validity of an epidemiologic transition in concurrence with new illnesses, the search of scientific and technological advances to answer the emergent demands and the adoption and consolidation of models on health prevention. The emphasis in new paradigms is a convergent point of the (inter)national official agencies agenda, namely the incentives to conduct research at biomedical and social sciences. Current public policies try to improve the quality of the lived years of the population, in an interdisciplinary, integrated, articulated and individualized perspective that covers the entire life course of the individual so that healthy life styles can be consolidated, with a special reference to the regular practice of physical activity.
\end{abstract}

Keyw ords: aging, public health, physical activity, healthy life styles

\section{O perfil mundial do envelhecimento}

A Organização Mundial de Saúde - OMS ou WHO, sigla anglófona, considerando o período correspondente ao ano de 1970 até 2025 (estimado), prevê um crescimento mundial de $223 \%$ no número de pessoas com $60+$ anos de idade. Com esta estimativa assegura que em 2025 existirão, aproximadamente, 1,2 milhar de milhões de pessoas com
60+ anos de idade e, em 2050 chegar-se-á aos dois milhar de milhões, dos quais $80 \%$ pertencerão aos países em desenvolvimento (WHO, 2005).

Nos países em desenvolvimento a dinâmica populacional tem apresentado um declínio menos acentuado nas taxas de mortalidade e de fecundidade, quando comparada com períodos anteriores, mas evidenciando já características próprias das populações pós transição demográfica. Segundo a 
OMS, um país é considerado estruturalmente enveIhecido quando a proporção de idosos ultrapassa $7 \%$ do total da população. Em demografia, os critérios normalmente utilizados para avaliar os ritmos de envelhecimento são a redução da proporção de jovens na base da estrutura etária e o aumento da proporção de pessoas idosas no topo da pirâmide. Tais fenómenos, quando analisados independentemente, possibilitam a aplicação das denominações: envelhecimento da base e envelhecimento do topo, respectivamente, uma vez que, na análise das estruturas etárias, tais fenómenos ocorrem de forma processual (Fernandes, 1997:31). Para o ano de 2025, a estimativa é de que 120 países terão alcançado taxas de fecundidade abaixo do nível de reposição geracional, ou seja, terão atingido uma capacidade de procriar inferior a 2,1 crianças por mulher, o limiar necessário para a reposição das gerações. (WHO, 2005).
Além dos dados apresentados a título de exemplo (Gráfico 1) podemos ainda destacar situações bem definidas como sejam: 1) nos países desenvolvidos, em 2002, havia aproximadamente 400 milhões de pessoas com $60+$ anos de idade, e uma estimativa de aumento de $110 \%$ até ao ano 2025 (70\% da população com mais de 60 anos, ao nível mundial); 2) na composição da população da União Europeia, a estimativa para 2050 prevê que três entre cada dez pessoas terão $65+$ anos de idade e que, particularmente para o Sul da Europa, 35,6\% da população espanhola, 35,3\% da italiana, 32,5\% da grega e $31,9 \%$ da população portuguesa terão mais de 65 anos em 2050 ; 3) mais da metade da população com $60+$ anos, quando analisadas as cinco regiões mundiais (Quadro 1 ), reside no Continente Asiático; 4) na América do Norte, as pessoas com $60+$ anos de idade passaram de $12,4 \%$ em 1950 para $16,7 \%$ em 2005 , com uma estimativa de

Gráfico $1 \triangleright$ Proporção de pessoas com 65+ anos, em alguns países a nível mundial, 2007.

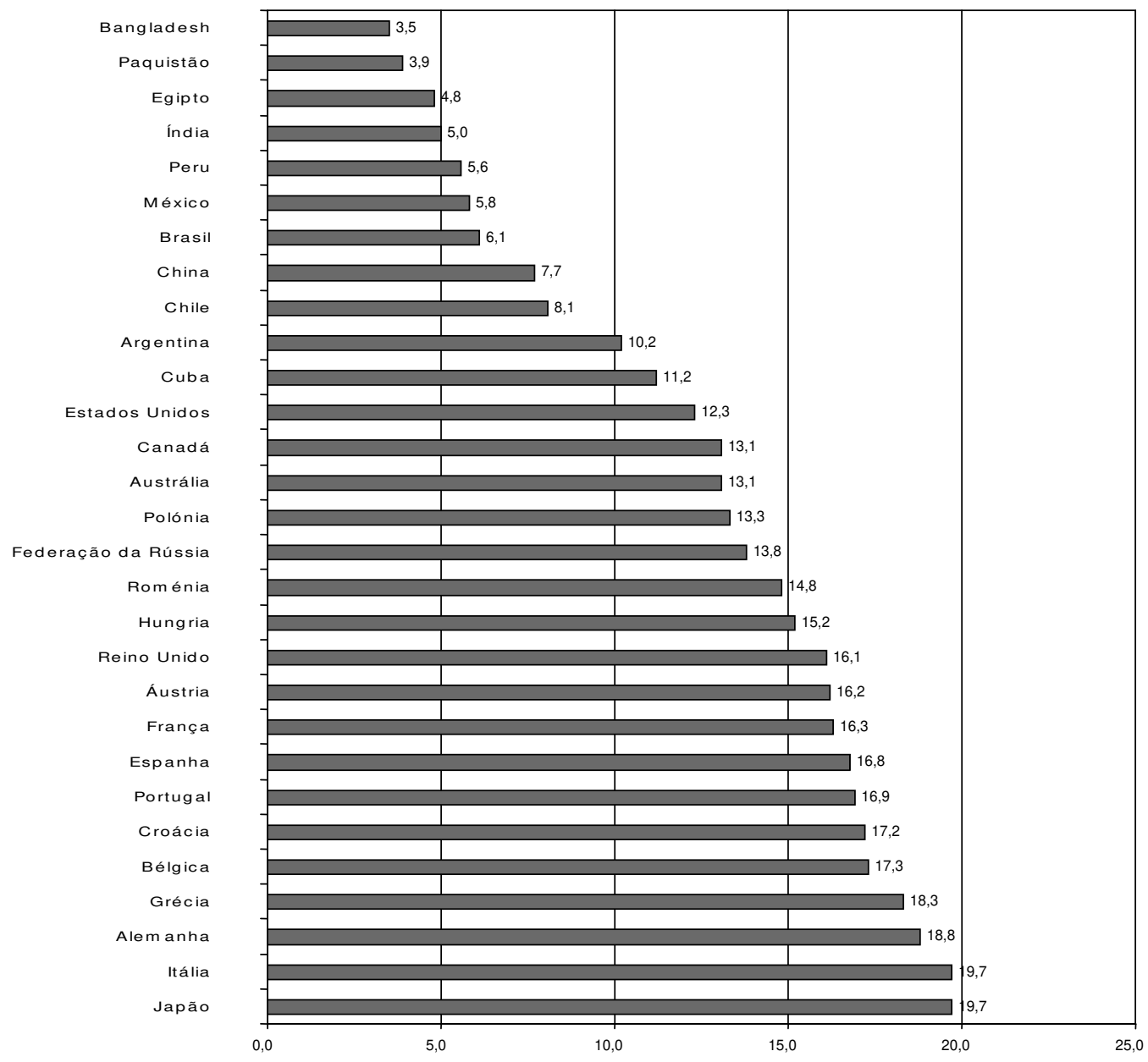

Fonte: UNDP, 2007. 
evolução para 20,1\% em 2015 e 27,3\% em 2050; 5) na América Latina e no Caribe, o grupo de 60 anos de idade ou mais constituía 5,6\% em 1950, aumentando para $9,0 \%$ em 2005, prevendo-se $24,3 \%$ em 2050, e, entre as pessoas com idade compreendida entre 60 e 64 anos de idade, $27,8 \%$ continuarão exercendo actividades laborais (Census Bureau, 2004; WHO, 2005; Allen, 2006; OPS, 2007a; OPS, 2007b). cimento Activo, um marco político para o processo de envelhecimento mundial, que destaca os direitos humanos das pessoas idosas quanto à independência, participação, dignidade, assistência e auto-realização. Este documento enfatiza a promoção da saúde, a prevenção de morbimortalidades, a melhoria da qualidade de vida, o alcance de níveis satisfatórios de atendimento nos sistemas de saúde e previdência social, valorizando e inserindo as pessoas idosas no

Quadro $1 \downarrow$ Distribuição da população mundial acima de 60 anos de idade, por região, em 2002 e projecção para o ano 2025

\begin{tabular}{|l|r|r|l|}
\hline \multicolumn{1}{|c|}{ Regiões } & 2002 & 2025 & \multicolumn{1}{c|}{ Tendência comparativa } \\
\hline África & $7 \%$ & $7 \%$ & Manutenção \\
\hline Ásia & $53 \%$ & $59 \%$ & Aumento \\
\hline América Latina e Caribe & $7 \%$ & $8 \%$ & Aumento discreto \\
\hline América do Norte & $8 \%$ & $8 \%$ & Manutenção \\
\hline Europa & $24 \%$ & $17 \%$ & Diminuição \\
\hline Oceania & $1 \%$ & $1 \%$ & Manutenção \\
\hline
\end{tabular}

Fonte: Nações Unidas apud World Health Organization, 2005a: 12

As considerações referentes ao envelhecimento da população têm ainda uma componente importante que é a transição epidemiológica relacionada com a morbilidade, invalidez e morte de uma população específica e que ocorre concomitantemente com outras alterações demográficas, sociais e económicas (Fernandes et al., 2004). No Brasil, como na América do Sul em geral, a transição está em curso, sendo caracterizada pela substituição das causas de morte, essencialmente de doenças transmissíveis por doenças não transmissíveis, e causas externas e de mortalidade em todas as idades, especialmente nas crianças, por morbilidade e mortalidade nas categorias etárias mais velhas. Nas Américas, as disfunções por enfermidades crónicas aumentaram $17 \%$, a diabetes aumentou em quase $80 \%$ (OPS, 2007a). Esta transição epidemiológica está associada ao aumento de problemas relacionados com a saúde da população idosa com predominância para as doenças crónicas e suas complicações (a exemplo, sequelas provenientes de acidentes vasculares celebrais; cancro e doenças músculo-esqueléticas; limitações provocadas por insuficiência cardíaca e doença pulmonar obstrutiva crónica; amputações e cegueira provocadas por diabetes; dependência decorrente da doença de Alzheimer; fracturas provenientes de quedas) (WHO, 2000; WHO, 2002; Brasil, 2002; WHO, 2007b; OPS, 2007a; Brasil, 2007a).

Ao estruturar uma política orientada para a população mundial em processo de envelhecimento, a OMS lançou, em 2002, o paradigma do Envelhe- contexto social e explicitando a actuação do Estado na consolidação deste paradigma (WHO, 2005).

\section{Perfil do Envelhecimento na População Brasileira}

Segundo a estimativa realizada pelas Nações Unidas para a população brasileira no ano de 2050 , o volume total será de 284 milhões de habitantes, sendo $17 \%$ jovens e $15 \%$ de pessoas com $60+$ anos de idade. Em 2000, o número de idosos com 60+ anos de idade, era de 14.536.029, ou seja, 8,6\% da população brasileira total (Brasil, 2000; Pessoa, 2007).

A caracterização da população idosa no Brasil - referimo-nos às pessoas com 60+ anos - e o seu impacto sobre a economia doméstica podem ser estimados pelo facto de $62,4 \%$ dos idosos serem responsáveis pelo seu domicílio $(37,6 \%$ do sexo feminino), com o seguinte perfil: $81 \%$ vivem na área urbana (havendo distribuição heterogénea: Rio de Janeiro - $12,8 \%$ e Porto Alegre - 11,8\% e Boa Vista - 3,8\% e Palmas - 2,7\%); as mulheres correspondem a $55,1 \%$ da população de idosos; os domicílios tinham em média 3,2 componentes e $17,9 \%$ eram domicílios unipessoais (dos quais $67 \%$ ocupados por mulheres); 64,8\% dos idosos estavam alfabetizados (média de 3,4 anos de estudo, variando de 6 anos no Distrito Federal a 1,5 anos no estado do Maranhão), havendo 5,1 milhões de idosos analfabetos. (Brasil, 2000; Brasil, 2002). 
Analisando as taxas da fecundidade e de mortalidade do Brasil ao longo do século XX, identificamos: 1) um declino acentuado na taxa de fecundidade, que se traduz pela redução do número total de filhos que uma mulher poderia ter no final do período reprodutivo: de 6,3 filhos (1960) para $2,3(2000) ; 2$ ) crescimento progressivo da esperança de vida, ao nascer, de 43,6 anos (1940) para 68,6 anos (2000); e 3) mudanças significativas no que respeita às causas de morte no período de 1980 a 2000, com relevância para o Programa Nacional Brasileiro de Doenças Sexualmente Transmissíveis/ /Síndrome da Imunodeficiência Adquirida (DST/ /SIDA) que possui impacto favorável sobre a redução das doenças infecciosas (Quadro 2) (Jannuzzi, 2001; MS, 2006). dados referentes às populações totais e de pessoas com 65+ anos de idade, à esperança de vida, aos valores dos IDHs, às taxas de escolaridade e de alfabetização (Quadro 3). (UNDP, 2007).

A OMS preconiza como factores determinantes da adopção de estilos de vida saudáveis: 1) a participação activa no cuidado da própria saúde; 2) o envolvimento em actividades físicas adequadas; 3 ) o consumo de alimentação saudável; 4) a abstinência tabágica; 5) o consumo moderado de álcool e 6) a utilização de medicamentos adequados (Laurenti et al.,2005; WHO, 2005).

Quando consideramos intervenções sobre os hábitos alimentares e a adesão a actividades físicas de uma população, é importante a adopção de estratégias para equilibrar o balanço energético

Quadro $2 \downarrow$ Ranking das principais causas de morte, Brasil. 1980 e 2000

\begin{tabular}{|c|c|c|c|}
\hline \multicolumn{2}{|r|}{ RANKING 1980} & \multicolumn{2}{|r|}{ RANKING 2000} \\
\hline Rank & Código CID 9 e Situações patológicas & Rank & Código CID 10 e Situações patológicas \\
\hline 1 & VII - Doenças do aparelho circulatório & 1 & VII - Doenças do aparelho circulatório \\
\hline 2 & $\begin{array}{l}\text { XVI - Sintomas, sinais e afecções mal } \\
\text { definidas }\end{array}$ & 2 & $\begin{array}{c}\text { XVI - Sintomas, sinais e achados anormais ex. clin } \\
\text { e laboratorial (mal definidas) }\end{array}$ \\
\hline 3 & XVII - Causas externas & 3 & II - Neoplasias (tumores) \\
\hline 4 & I - Doenças infecciosas e parasitárias & 4 & $\begin{array}{l}\text { XX - Causas externas de mobilidade e mortali- } \\
\text { dade }\end{array}$ \\
\hline 5 & II - Neoplasias & 5 & X - Doenças do aparelho respiratório \\
\hline 6 & VIII - Doenças do aparelho respiratório & 6 & $\begin{array}{l}\text { IV - Doenças endócrinas, nutricionais e metabóli- } \\
\text { cas }\end{array}$ \\
\hline 7 & 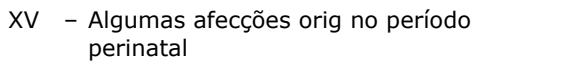 & 7 & I - Algumas doenças infecciosas e parasitárias \\
\hline 8 & $\begin{array}{l}\text { III - Glând. Endócrinas, nutricionais, metab. e } \\
\text { transtornos imunitários }\end{array}$ & 8 & XI - Doenças do aparelho digestivo \\
\hline 9 & IX - Doenças do aparelho digestivo & 9 & XVI - Algumas afec. originadas no período perinatal \\
\hline 10 & Sistema nervoso e órgãos dos sentidos & 10 & XIV - Doenças do aparelho geniturinário \\
\hline
\end{tabular}

Fonte: MS - Ministério da Saúde, Brasil, 2004.

\section{A actividade física em programas de promoção da saúde}

O facto de o Programa das Nações Unidas para o Desenvolvimento (PNUD) calcular o índice de desenvolvimento humano - IDH e considerá-lo como critério a ser aplicado em todos os Estados-membros das Nações Unidas permite uma análise comparativa. Quando, apurando o IDH e outros indicadores relevantes cotejamos os países que compõem o G-13 (ou seja, as sete nações mais industrializadas do mundo: Estados Unidos da América, Japão, Alemanha, Reino Unido, França, Itália e Canadá; a Federação Russa e os cinco países em desenvolvimento: China, Índia, México, Brasil e África do Sul), observamos importantes disparidades nos da procura individual e para ajustar a manutenção do peso corporal segundo a estatura, o biótipo e a configuração óssea, o que se irá reflectir na qualidade de vida nos anos subsequentes. O balanço energético traduzirá a proximidade ou o distanciamento do equilíbrio entre a ingestão e o gasto energético, constituindo-se em parâmetro norteador capaz de conciliar necessidades calóricas, gastos energéticos, hábitos sociais e estado emocional, além de assegurar uma taxa metabólica basal a partir da metabolização dos alimentos e disponibilizar reservas energéticas (Boule et al., 2001; Themudo-Barata, 2003; WHO, 2006; UNDP, 2007; WHO, 2007a).

Há evidência de que a relação entre os factores de risco da morbimortalidade, principalmente entre as doenças não transmissíveis - os factores ambientais 
Quadro $3 \triangleright$ Valores e classificação do índice de desenvolvimento humano; população total e de 65 anos de idade ou mais estimada para os anos de 2005 e 2015; esperança de vida em anos, taxa de alfabetização e PIB per capita do G13 (grupo dos treze)

\begin{tabular}{|c|c|c|c|c|c|c|c|c|c|}
\hline PAÍS & Valor IDH & $\begin{array}{c}\text { Ordem } \\
\text { IDH }\end{array}$ & $\begin{array}{l}\text { Pop total } \\
2005\end{array}$ & $\begin{array}{l}\text { Pop total } \\
2015\end{array}$ & $\begin{array}{c}\text { Pop } \geq 652005 \\
(* *)\end{array}$ & $\begin{array}{l}\text { Pop. } \geq 65 \\
2015(* *)\end{array}$ & $\begin{array}{c}\text { Esperança } \\
\text { de Vida } 2005\end{array}$ & $\begin{array}{c}\text { Taxa de } \\
\text { alfabetização } \\
(* * *)\end{array}$ & $\begin{array}{c}\text { PIB } \\
\text { per capita } \\
\text { (US\$) }\end{array}$ \\
\hline Estados Unidos & 0,951 & 12 & 299,8 & 329,0 & 12,3 & 14,1 & 77,9 & 99,0 & 41,890 \\
\hline Japão & 0,953 & 8 & 127,9 & 126,6 & 19,7 & 26,2 & 82,3 & 99,0 & 31,267 \\
\hline Alemanha & 0,935 & 22 & 82,7 & 81,8 & 18,8 & 20,9 & 79,1 & 99,0 & 29,461 \\
\hline Reino Unido & 0,946 & 16 & 60,2 & 62,8 & 16,1 & 18,1 & 79,0 & 99,0 & 33,238 \\
\hline França & 0,952 & 10 & 61,0 & 63,7 & 16,3 & 18,5 & 80,2 & 99,0 & 30,386 \\
\hline China & 0,777 & 81 & $1.313,0$ & $1.388,6$ & 7,7 & 9,6 & 72,5 & 90,9 & 6,757 \\
\hline Itália & 0,941 & 20 & 58,6 & 59,0 & 19,7 & 22,1 & 80,3 & 98,4 & 28,529 \\
\hline Canadá & 0,961 & 4 & 32,3 & 35,2 & 13,1 & 16,1 & 80,3 & 99,0 & 33,375 \\
\hline Índia & 0,619 & 128 & $1.134,4$ & $1.302,5$ & 5,0 & 5,8 & 63,7 & 61,0 & 3,452 \\
\hline México & 0,829 & 52 & 104,3 & 115,8 & 5,8 & 7,5 & 75,6 & 91,6 & 10,751 \\
\hline Brasil & 0,800 & 70 & 186,8 & 210,0 & 6,1 & 7,7 & 71,7 & 88,6 & 8,402 \\
\hline Rússia & 0,802 & 67 & 144,0 & 136,5 & 13,8 & 13,1 & 65,0 & 99,4 & 10,845 \\
\hline África do Sul & 0,674 & 121 & 47,9 & 50,3 & 4,2 & 5,5 & 50,8 & 82,4 & 11,110 \\
\hline
\end{tabular}

Fonte: UNDP, Relatório de Desenvolvimento Humano 2007

Nota dos autores: $\mathrm{IDH}=$ índice de desenvolvimento humano; $(*)=$ população total por milhões de habitantes; (**) População de $\geq 65$ anos de idade em \% do total da população; (***) Taxa de alfabetização de adultos com 15 anos de idade ou mais em percentagem (***)

(ambientes de fumo, agentes infecciosos, radiação, produtos químicos, industriais e poluição, entre outros) e comportamentais (consumo do tabaco, álcool e medicamentos, etc.) - têm maior impacto na morbilidade do que as determinantes de origem genética ou hereditária. Devido ao quantitativo mundial de casos de cancro (58 milhões de mortes em 2005 com estimativa de 15 milhões casos/ano para 2020) e ao desenvolvimento do conhecimento da biologia no desencadear da doença, é sabido que os hábitos alimentares, a inactividade física e a composição corporal são factores de risco relevantes para a situação mencionada passíveis de prevenção. (WCRF/AICR, 2007).

Apesar da urbanização, da facilidade na utilização de transporte motorizado, da expansão de políticas urbanas que privilegiam o uso de automóveis, da restrição no tempo livre das pessoas, da maximização das actividades intelectuais e sedentárias, da divulgação de actividades recreativas sedentárias, a prática de actividades físicas tem sido fortemente implementada ao nível mundial através de dispositivos baseados na presunção de elevados benefícios para a saúde pública. Na Europa há evidências da associação da prática de actividades físicas com a redução de risco em certo tipo de doenças (doenças cardíacas; síndromes de má perfusão; aumento de peso/obesidade; diabetes tipo 2; cancro do cólon; cancro cerebral; quedas de pessoas idosas e depressão) (Robine et al., 2001; WHO, 2006).

Na China, a Lei de Saúde Pública, instituída em 1995 , possibilitou que $33,9 \%$ dos chineses frequentassem clubes desportivos e se inserissem em actividade física, em 2000, e em 2005 se estendesse a $40 \%$ a percentagem de chineses que praticam pelo menos uma modalidade de actividade diária (State Council of the People's Republic of China, 2006).

No continente americano, o Plano Estratégico para as Américas 2008-2012 exprime a preocupação com os baixos níveis de actividade física. No Brasil, a prevalência da inactividade física em adultos - a média em 16 capitais brasileiras é de $37 \%$ em 2002/2003, variando em Belém (28\%) a João Pessoa (55\%) - é motivo de política pública governamental para contrariar esta tendência (Buss, 2006).

A propósito das contribuições da actividade física para o envelhecimento activo é enfatizada a necessidade de: 1) desenvolver informações e directrizes culturalmente apropriadas sobre actividade física baseadas no perfil da população e ajustada ao género; 2) fornecer oportunidades acessíveis, de baixo custo e agradáveis para que todos os idosos permaneçam activos; 3) apoiar grupos e líderes que promovam actividade física regular e moderada para pessoas durante o processo de envelhecimento e 4) informar e treinar os indivíduos e profissionais para a importância de permanecerem activos enquanto envelhecem (WHO, 2005). A adopção de níveis satisfatórios e adequados de actividade física de intensidade moderada, praticada regularmente durante o maior número de dias da semana (no mínimo três), com duração em torno de 30 minutos, com a inclusão de etapas onde ocorram exercícios de resistência muscular, equilíbrio, agilidade e flexibilidade, contribui para o controle do peso e para a redução do risco de doenças cardiovasculares, diabetes, 
cancro do cólon, além de aumentar a capacidade funcional e a mobilidade, melhorar o perfil lipidico, prevenir doenças do aparelho músculo-esquelético e o risco de quedas, lesões e fracturas associadas, especialmente nos mais velhos. As respostas ao treino de endurance e força, por indivíduos idosos, mantêm e melhoram os níveis de volume de oxigénio $\left(\mathrm{VO}_{2}\right)$ máximo, débito cardíaco e diferença arterio-venosa de 02, ajudando a compensar a redução na massa e força muscular tipicamente associada ao processo de envelhecimento. (Oliveira, 2005; WHO, 2006; Edwards \& Tsouros, 2006; Bennett et al., 2006; Lindwall et al., 2006; WHO, 2007a; Nelson et al., 2007).

Para clarificar a compreensão da actividade física no contexto do processo do envelhecimento, destacamos sete conceitos, a saber: 1 ) actividade da vida diária (AVD) - "capacidade para desempenhar tarefas físicas e actividades de cuidados pessoais mais básicas" (Katz et al., 1963:917); 2) actividades instrumentais da vida diária (AIVD) - "capacidade para desempenhar as tarefas necessárias ao funcionamento em casa ou na comunidade" (Fillenbaum \& Smyer, 1981:429), 3) capacidade funcional - condição para "manter as habilidades físicas e mentais necessárias para uma vida independente e autónoma, permitindo a realização das actividades básicas e instrumentais da vida diária" (Freitas, et al., 2002:865); 4) autonomia - entendida como a "actividade de controlar, lidar e tomar decisões pessoais sobre como se deve viver diariamente, de acordo com suas próprias regras e preferências" (WHO, 2005:6); 5) exercício - "actividade de tempo regular, padronizada, que procura alcançar resultados de aptidão desejáveis, melhorando o nível de saúde geral ou desempenho físico" (Bouchard \& Shephard, 1994:183); 6) actividade física - "qualquer actividade que intensifique o gasto energético acima daquele consumido durante o repouso e que resulte num movimento voluntário que cause a contracção muscular, podendo ser exemplificado pelo acto de andar, dançar, correr, pedalar, subir e descer escadas, nadar ou realizar jardinagem" (Matsudo et al., 2005:162) e 7) aptidão física - concebida como a "habilidade de suportar as tarefas diárias com vigor e atenção, sem fadiga exagerada e com ampla energia para aproveitar os períodos de folga e ainda ser apto a enfrentar situações de emergências previstas" (Clarke, 1976:102).

Suportes teóricos acrescidos do treino desportivo podem ser aplicados com êxito na prescrição de actividades físicas ao longo do ciclo da vida classificados, entre outros, pelos princípios científicos da: 1) individualidade biológica - prevê o respeito pelas peculiaridades de cada indivíduo como um ser único, que possui estrutura física e psicológica próprias, além de conciliar genótipo e fenótipo com as aptidões e habilidades; 2) adaptação - assegura que haja uma transição gradual e segura dos níveis actuais de actividade ou de sedentarismo para níveis de maior sobrecarga do exercício ou da actividade propriamente dita (exercícios físicos, actividades recreativas ou desportivas); 3) sobrecarga - enfatiza a necessidade de se realizar uma sobrecarga maior do que aquela a que se está habituado para que haja uma resposta fisiológica ao treino físico, sendo esta sobrecarga controlada pela intensidade, duração e frequência da actividade física; 4) especificidade baseia-se no facto de que diferentes modalidades de actividade física desencadeiam adaptações que promovem respostas fisiológicas específicas; 5) reversibilidade - faz com que as adaptações fisiológicas promovidas pela realização de actividade física retornem ao estado original de pré-treino quando o indivíduo volta ao estilo de vida sedentário (Matveev, 2001). Especificamente em relação aos idosos, a classificação da capacidade funcional de Spirduso, 1989, fornece parâmetros globais para avaliação no que concerne aos: 1) idosos fisicamente dependentes; 2) idosos fisicamente frágeis; 3 ) idosos fisicamente independentes; 4) idosos fisicamente activos; e 5) idosos atletas.

Muitas actividades físicas têm sido utilizadas para avaliação de uma pessoa em processo de envelhecimento (Quadro 4) com destaque para o Questionário Internacional de Actividade Física (IPAQ), em fase de validação internacional, que tem por finalidade disponibilizar dados internacionais por meio de um instrumento de medida do nível de actividade física que permita comparar resultados nos níveis regionais, nacionais e internacionais (Matsudo Et Al., 2001; Morrow et al., 2003, Craig et al., 2003; Armstrong \& Bull, 2006).

Em consonância com a preocupação mundial de consolidar uma estratégia global de dieta, actividade física e saúde capaz de incluir recursos e investimentos (inter)nacionais e que preencha a lacuna existente entre o sector da saúde e sectores afins (nomeadamente: educação, planeamento urbano, transporte, comunicação) a OMS publicou um guia para a implementação de políticas crescentes de actividade física, estabelecendo directrizes para maximizar níveis adequados de saúde estruturados na actividade física, ao longo do ciclo da vida. Entre os objectivos de tal política destacamos: a prevenção e controle das doenças crónicas e não transmissíveis; a aquisição de melhores condições de saúde e bemestar físico, mental e social e não somente visando a valorização da autonomia e independência além da simples ausência de doenças (Lebrão e Duarte, 2003; WHO, 2007b, Pessoa, 2007).

Diante do exposto, e considerando que o aumento da esperança de vida origina o alargamento das categorias no topo da pirâmide etária com mais anos de vida saudáveis, mudanças estruturais nas relações familiares e de trabalho, a emergência de 
Quadro $4 \triangleright$ Lista de actividades físicas utilizadas para avaliação de uma pessoa em processo de envelhecimento

\begin{tabular}{|l|l|l|}
\hline Agachar-se e/ou pegar objecto no chão & Deitar-se e levantar-se & Sentar/levantar e caminhar para casa \\
\hline Arrumar a cama & Entrar e sair do carro & Subir e descer degraus de transporte \\
\hline Actividades manuais & Executar higiene pessoal & Subir e descer escadas \\
\hline Calçar meias & Executar força de preensão & Subir e descer cadeiras \\
\hline Caminhar distâncias médias & Lavar louça & Executar tarefas manuais \\
\hline Carregar pesos & Lavar roupa & Tomar banho \\
\hline Correr & Levantar-se do solo & Varrer a casa \\
\hline Cozinhar & Permanecer em pé & Vestir-se \\
\hline
\end{tabular}

Fonte: Katz et al.,(1963); Kuriansky et al., (1980); Clark (1989); Reuben \& Siu (1990); Voorrips et al., (1991); Riki \& Jones (1999); Riki \& Jones (2000); Botelho (2000).

novas regras no mercado de trabalho, taxas de inactividade em ritmo crescente e aumento da procura de cuidados de saúde e de protecção social, entre outros efeitos) (Campos, et al., 2004), corroboramos o alerta feito por Anne-Marie Guillemard:

"(...) há necessidade de um novo paradigma que contemple um novo desenho de direitos sociais nas relações do trabalho (o único modelo com normas está a desaparecer) que seja capaz de converter a flexibilidade de tempo imposta pelo mundo da produção numa liberdade gerida por trabalhadores individuais, aumentando para todos a compatibilidade entre os diferentes aspectos da vida - trabaIho com remuneração, o trabalho gratuito, a formação, o lazer, o tempo com a família e para descansar - e que permita a cada pessoa exercer controle sobre o seu tempo e a sua vida." (Guillemard, 2001: 7-8)

\section{Considerações Finais}

Considerando que o processo de envelhecimento de um país ocorre quando a proporção de idosos ultrapassa $7 \%$ do total de sua população e que há estimativas para que 120 países, em 2025, tenham atingido uma fecundidade de 2,1 crianças por mulher, com taxas de fecundidade abaixo do nível de reposição, fica evidente que o processo de envelhecimento é um processo global que terá impacto sobre a situação socio-económica e cultural da população. O facto de a transição epidemiológica constituir um evento de deslocação das causas de morte e se repercutir no perfil de morbilidade, de grupos mais jovens para grupos mais idosos, contribui para avaliar a magnitude do envelhecimento da população como um processo mundial.

O Envelhecimento Activo representa um marco político mundial, ao acentuar a importância da promoção da saúde no processo de envelhecimento com destaque para a participação e integração, dignidade, auto-realização, autonomia, ambientes seguros e protecção e suporte nas situações de doença e incapacidade, em contexto familiar e comunitário. A prática de actividade física insere-se num conjunto de factores que contribuem para a manutenção e melhoria das condições de saúde e bem-estar das pessoas que envelhecem e é uma dos determinantes mais poderosas para proporcionar um envelhecimento saudável e activo.

\section{Referências bibliográficas}

ARMSTRONG, T. e F. Bull (2006), «Development of the World Health Organization Global Physical Activity Questionnaire (GPAQ)», Journal of Public Health, v.14, n², pp. 66-70.

BENNETT, J.A., K. Winters-Stone, L.M. Nail e J. Scherer (2006), «Definitions of sedentary in physicalactivity-intervention trials: a summary of the literature», Journal of Aging and Physical Activity, v.14, pp. 456-477.

BOTELHO, M.A.S. (2000), «Autonomia Funcional em Idosos: caracterização multidimensional em idosos utentes de um centro de saúde urbano», Tese de Doutoramento, Faculdade de Ciência Médicas da Universidade Nova de Lisboa, Lisboa, pp.1-258.

BOUCHARD, C. e R.J. Shephard (1994), «Physical activity, fitness, and health: International proceedings and consensus statement», Human Kinetics Publishers, Illinois, pp. 180-199.

BOULE, N.G., E. Haddad, G.P. Kenny, G.A. Wells e R.J. Sigal (2001), «Effects of exercise on glycemic control and body mass index in type II diabetes: a meta-analysis of controlled clinical trials», JAMA, v. 286,10 , pp.1218-27.

BRASIL (2000), Fundação Instituto Brasileiro de Geografia e Estatística (IBGE), «Censo 2000», Disponível em: <www.ibge.gov.br> acedido em 20 jan 2006 às 21:00 horas, Brasilia, DF.

BRASIL (2007a), Fundação Instituto Brasileiro de Geografia e Estatística, "Tabela das estimativas das 
populações», Disponível em <Ftp://ftp.ibge.gov. br/Estimativas_Projecoes_Populacao/>.

BRASIL (2002), Relatório Nacional sobre Envelhecimento Populacional, Brasília, DF.

BUSS, P.M. (2006), «National strategy on diet, physical activity and health in Brazil», Oswaldo Cruz Foundation, Secretaria de Vigilância em Saúde, Brasil, pp.1-18.

CAMPOS, G.W., R.B. Barros e A.M. Castro (2004), Avaliação de política nacional de promoção da saúde, Ciência e Saúde Coletiva, Rio de Janeiro, v.9, no 3, pp.745-749,

CENSUS BUREAU, 2004, Estados Unidos da América, «US Interim projections by age, sex, race, and hispanic origin», 2004. Disponível em <http:// www. census.gov/lpc/www/usinterimproj/> Acedido em 29/10/2006 às 13:12horas.

CLARKE, H.H. (1976), Application of measurement to health and physical education, Prentice Hall Inc., New Jersey, pp.446.

CLARK, B.A. (1989), «Tests for fitness in older adults: AAHPERD Fitness Task Force», Journal of Physical Education, Recreation and Dance, v. 3, pp. 66-71.

CRAIG, C.L., A.L. Marshall, M. Sjöström, A.E. Bauman, M.L. Booth, B.E. Ainsworth, M. Pratt, U. Ekelund, A. Yngve, J.F. Sallis e P. Oja (2003), "International Physical Activity Questionnaire: 12 country reliability and validity", Med Sci Sports Exerc., August, v.35, 8, pp.1381-1395.

EDWARDS, P. e A. Tsouros (2006), "Promoting physical activity and active living in urban environments", The Regional Office for Europe of the Worls Health Organization, pp.1-66.

FERNANDES, A.A. (1997), Envelhecimento e saúde: demografia, família e políticas sociais em Portugal, Celta Editora, Oeiras, pp.1-211.

FERNANDES, A.A., M.J.G. Moreira e T.R. Veiga (2004), "Transição Demográfica e Transição Epidemiológica. O modelo de mortalidade no contexto das transformações sociais e políticas da sociedade portuguesa", Fórum Sociológico, 11/12 (2a. Série), pp. 75-104.

FILLENBAUM, G.G, M.A. Smyer (1981), «The development, validity and reabiability of the OARS multidimensional functional assessment questionnaire», Journal of Gerontology, v. 36, pp.428-434.

FREITAS, E.V., L. Py, N.A. Liberalesso, F.A.X. Cançado, M.L. Gorzoni e S.M. Rocha (2002), Tratado de Geriatria e Gerontologia, Ed. Guanabara Koogan, São Paulo/Rio de Janeiro/Belo Horizonte, pp.1-1187.

GUILLEMARD, A.M. (2001) "The advent of a flexible life course and the reconfiguration of welfare", Conference Social Policy, Marginalisation, and Citizenship, Aalborg University, Denmark 2-4, pp1-13.

JANNUZZI, P.M. (2001), Indicadores sociais no Brasil: conceitos, fontes de dados e aplicações de políticas públicas, elaboração de estudos econômicos, Alínea, Campinas, pp.1-141.
KATZ, S., A.B. Forod, R.W. Moskowitz, B.A. Jackson, B.A. e M.W. Jaffe (1963), «Studies of illness in the aged: the index of ADL a standardized measure of biological and psychosocial function», Journal of the American Medical Association, v.185, 12, pp. 914-919.

KURRIANSTY, J. B., A. Rundgren e L. Sperling (1980), «Evaluation of functional capacity in activities of daily living in 70-year-old men and women. Scandinavian», Journal of Rehabilitation Medicine, v.12, pp. 145-54.

LAURENTI, R., C.M. Buchalla, J.M.H.P. Mello, M.L. Lebrão, S.L.D. Gotlieb (2005), «Perfil epidemiológico da saúde masculina na Região das Américas: uma contribuição para o enfoque de gênero», Ciência Saúde Coletiva jan/mar, v.10, 1, pp. 35-46.

LEBRÃO, M.L. e Y.A.O. Duarte (2003) (orgs.), SABE Saúde, Bem-estar e Envelhecimento - O Projeto Sabe no município de São Paulo: uma abordagem inicial, Organização Pan-Americana da Saúde, Brasília, pp. 255.

LINDWALL, M., M. Rennemark, A. Halling, J. Berglung e P. Hassmen (2006), "Depression and exercise in elderly men and women: findings from the Swedish National Study on aging and care", Journal of Aging and Physical Activity, v.15, pp. 41-55.

MATSUDO, S., T. Araújo, V. Matsudo, D. Andrade, E. Andrade, L.C. Oliveira e G. Braggion (2001), "Questionário internacional de atividade física (IPAQ): estudo de validade e reprodutibilidade no Brasil, São Caetano do Sul", Revista Brasileira de Atividade Física e Saúde, v.6, n², pp. 05-18.

MATSUDO, V.V.K.R., S.M.M. Matsudo, T.L. Araujo e M.A. Ribeiro (2005), "Dislipidemias e a promoção da atividade física: uma revisão na perspectiva de mensagens de inclusão", Revista Brasileira de Ciência e Movimento, v.13, n², pp. 161-170.

MATVEEV, L.P. (2001), Teoría general del entrenamiento deportivo, Paidotribo, Barcelona, pp. 1-255.

MS - Ministério da Saúde (2006), "A contribuição dos estudos multicêntricos frente à epidemia de HIV/ Aids entre UDI no Brasil: 10 anos de pesquisa e redução de danos", Ministério da Saúde, Brasília.

MORROW, J.R., A.W. Jackson, J.G. Disch e D.P. Mood, (2003), Medida e avaliação do desempenho humano, $2^{\mathrm{a}}$ ed. Trad. Maria da Graça Figueiró da Silva, Artmed, Porto Alegre, p.1-304.

NELSON, M.E, W.J. Rejeski, S.N. Blair, P.W. Duncan, J.O. Judge, A.C. King, C.A. Macera e C. Castaneda -Sceppa (2007), "Physical activity and Public Health in Older Adults: recommendation from the American College of Sports Medicine and the American Heart Association", Medicine and Science in Sports and Exercise, v.39, 8, pp. 1435-1445.

OLIVEIRA, A.C.B. (2005), "Estudo comparativo dos efeitos da atividade física com os da terapêutica medicamentosa em idosos com depressão maior", Tese de doutoramento, Faculdade de Medicina da Universidade de São Paulo, São Paulo, Brasil, pp.1-102. 
OPS - Organización Panamericana De La Salud (2007a), Agenda de la Salud para las Américas 2008-2017, Panamá, OPS, pp.1-864.

OPS - Organización Panamericana De La Salud (2007b), Propuesta del Plan Estratégico 2008-2012, Documento oficial 328, OPS, pp.1-151.

PESSOA, I. L. (2007), "As Políticas de saúde destinadas à pessoa idosa no Brasil frente ao fenômeno do envelhecimento populacional", XIII Congresso Brasileiro de Sociologia, UFPE, Recife/PE, Brasil.

REUBEN, D.B. e A.L. Siu (1990), "Objective measure of physical function of elderly outpatients: the physical performance test", Journal of the American Geriatrics Society, v.38, n¹0, pp.1105-12.

RIKLI, R.E. e C.J. Jones (1999), "Development and validation of a functional fitness test for community-residing older adults", Journal of Aging and Physical Activity, v.7, pp.129-61.

RIKLI, R.E. e C.J. Jones (2000), "Reliability, validility, and methodological issues in assessing physical activity in older adults", Research Quaterly for Exercise \& Sport, v.71, pp. 89-96.

ROBINE, J.M., C. Jagger, A. Clavel, e I. Romieu (2001), "Disability-free life expectancy (DFLE) in EU countries from 1991 to 2003", European Health Expectancy Monitoring Unit (EHEMU), v.1, pp.1-25.

SPIRDUSO, W.W. (1995), Physical dimensions of aging, Human Kinetics, Champaign, pp1-432.

STATE COUNCIL OF THE PEOPLE'S REPUBLIC OF CHINA (2006), "«The Development of China's Undertakings for the Aged"», Beijing. Disponível em: <http://www.china.org.cn/english/aged/192020. htm> acedido em 20 dez 2007 às 21:00 horas.

THEMUDO-BARATA, J.L. (2003), Mexa-se ... pela sua saúde: guia prático de actividade física e emagrecimento para todos, Publicações Dom Quixote, Lisboa, pp.1-250.

UNDP - UNITED NATIONS DEVELOPMENT PROGRAMME (2007), Human Development Report 2007/2008,
Fighting Climate Change: Human Solidarity in a Divided World, Palgrave Macmillan, New York, pp.1-402.

VERAS, R.P (1994), País jovem com cabelos brancos: a saúde do idoso no Brasil, Relume Dumará, Rio de Janeiro, pp.1-213.

Voorrips, L.E., A.C.J. Ravelli, P.C.A. Dongelmans, P. Deurenberg e W.A.V. Staveren (1991), "A physical questionarie for the elderly", Medicine and Science in sports and Exercise, v.23, n०8, pp.979-9.

WCRF/AICR- WORLD CANCER RESEARCH FUND/ /AMERICAN INSTITUTE FOR CANCER RESEARCH (2007), Food, Nutrition, Physical Activity, and the Prevention of Cancer: a Global Perspective, DC, AICR, Washington, pp. 1-537.

WHO - World Health Organization (2007a), "A guide for population-based approaches to increasing levels of physical activity: implementation of the WHO global strategy on diet, physical activity and health", v.I.WHO, Geneva, pp.1-25.

WHO - World Health Organization (2007b), "Healthy ageing, a keystone for a Sustainable Europe", DG ECFIN and DG EMPL, January, pp.1-22.

WHO - World Health Organization (2006), "A framework to monitor and evaluate the implementation of the WHO Global Strategy on Diet, Physical Activity and Health", WHO, Geneva, pp.1-32.

WHO - World Health Organization (2005a), "Envelhecimento ativo: uma política de saúde", Organização Pan-Americana da Saúde, Trad. Organização Mundial de Saúde, Brasília, pp.1-14.

WHO - World Health Organization (2002), "The World Health Report, Reducing risks: promoting healthy", WHO Technical Report, Geneva, pp.1-13.

WHO - World Health Organization (2000), "Obesity: preventing and managing the global epidemic", Report of a WHO Consultation, WHO Technical Report Series 894, Geneva, pp. 1-265 